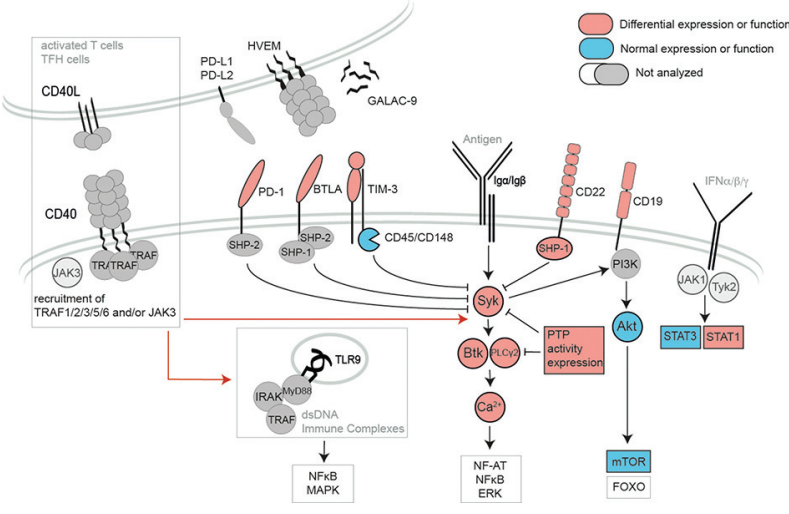

Abstract SP0084 Figure 1. Functiotypic characteristics of post-activated B cells from autoimmune patients.

Disclosure of Interests: None declared

DOI: 10.1136/annrheumdis-2019-eular.8527

THURSDAY, 13 JUNE 2019

15:30:00 - 17:00:00

Fracture liaison service: an opportunity for rheumatologists to focus on secondary fracture prevention

\section{SP0085 WHOM TO IDENTIFY AND INVITE TO AN FLS? WHO IS THAT FRACTURE PATIENT?}

\section{Bo Abrahamsen. $x, x, x$, Denmark}

Background: Though osteoporosis can be diagnosed through the presence of fragility fractures in the absence of other aetiology such as myeloma or metastases, patients with osteoporosis are also at increased risk of high impact traumatic fractures. Further, distinguishing between fracture mechanisms through chart review is often difficult and may lead to patients with osteoporosis being missed. On the other hand, focusing on identifying and treating patients at high imminent fracture risk rather than milder degrees of osteoporosis makes for better cost utility.

Objectives: To review the demographics of patients presenting with fragility fractures and the evidence for targeting osteoporosis assessment for each category of fracture in order to balance the need for maximum risk reduction with the requirement for an evidence based strategy.

Methods: Narrative review of recent epidemiology studies and national and international guidelines

Results: Based on epidemiology data from Iceland(1) and Denmark(2,3), the risk of subsequent fractures following a sentinel fracture event is critically dependent on the recency and the site of the initial fracture, with the risk of new fractures being particular high after major osteoporotic fractures(1) and pelvic fractures but less so after lower leg fractures(2).

In Danish women(2), 29.5\% of patients suffering a pelvic fracture went on to sustain a hip fracture in the next ten years, compared with $25.9 \%$ after a vertebral fracture but only $12.5 \%$ after a lower leg fracture. Further, despite the high recurrent fracture risk in the FLS setting, it is important to appreciate that the majority of hip fracture patients have not consulted with a prior fracture in the last ten years prior to their hip fracture(3).

The following issues will be addressed in more detail

1) Demographics of fracture patients with particular emphasis on age, sex and BMD.

2) Which fracture types are indicators of elevated risk of subsequent major osteoporotic fractures?

3) Which fracture types will respond to osteoporosis treatment?

4) What is the role of DXA in FLS?

5) Identifying vertebral fractures

Conclusion: FLS patients are at elevated risk of sustaining additional fractures both in the long term and in the short term, with risks being particularly high in the first years after the sentinel fracture and especially if the initial fracture is a pelvic fracture or a major osteoporotic fracture.

\section{REFERENCES:}

[1] Kanis, Osteoporos Int 2018, 29:1747-1757
[2] Hansen, Osteoporos Int 2015, 26:513-519

[3] Frederiksen, Osteoporos Int 2018; 29:109-114

Disclosure of Interests: None declared DOI: 10.1136/annrheumdis-2019-eular.8411

\section{SP0086 ASSESSMENT: HOW TO PERSONALIZE SECONDARY FRACTURE PREVENTION?}

Daniel Prieto-Alhambra. University of Oxford, NDORMS, Oxford, United Kingdom

Background: The existing armamentarium of therapeutic options to minimise fracture risk is growing. Different therapies have different properties, and can be used for different patient targets.

Objectives: To discuss data on short-term fracture risk following an index fracture, and on the short-term efficacy of different therapies.

Methods: Narrative review of recent literature.

Disclosure of Interests: Daniel Prieto-Alhambra Grant/research support from: Grants from Amgen, UCB Biopharma and Servier outside the submitted work, Consultant for: UCB Biopharma, Speakers bureau: Amgen DOI: 10.1136/annrheumdis-2019-eular.8564

\section{SP0087 TREATMENT: HOW TO IMPLEMENT IT AND HOW TO MONITOR ADHERENCE?}

Kassim Javaid Muhammad. University of Oxford, Nuffield Department of Orthopaedics, Rheumatology and Musculoskeletal Sciences, oxford, United Kingdom

Background: The key to achieving the benefits of an FLS on the population and the patient level is to ensure enough high-risk patients are initiated and then adhere to therapy. There is a low rate of primary and secondary adherence by patients with osteoporosis. Treatment initiation requires co-developed decision making with patients and clinicians balancing locally agreed intervention thresholds with effective and efficient risk and treatment communication. Treatment adherence depends on patient, treatment and healthcare service characteristics. Objectives: Describe the current status of treatment recommendation in the UK Describe the current methods for imrpoving adherence globally

Methods: Review of the data from the UK Fracture Liaison Service Database and IOF Capture the Fracture audits.

Disclosure of Interests: Kassim Javaid Muhammad Shareholder of: Zebra Medical Vision, Grant/research support from: Amgen, Kyowa Kirin Hakin, Consultant for: Amgen, Internis, Consilient Health,Mereo Biopharma, Kyowa Kirin Hakin, UCB, Speakers bureau: Amgen, Lilly UK, Internis, UCB

DOI: 10.1136/annrheumdis-2019-eular.8508

\section{THURSDAY, 13 JUNE 2019}

15:30:00 - 17:00:00

\section{How not to smoke like a chimney}

\section{SP0088 HOW YOU KNOW IT IS SMART NOT TO SMOKE LIKE A CHIMNEY}

Antonio Naranjo. Hospital Universitario de Gran Canaria Dr Negrin, Rheumatology, Las Palmas de Gran Canaria, Spain

Smoking is associated with a higher prevalence of many diseases. When a health professional is well informed of the risks of smoking in rheumatic diseases, the advice given to the patient about quitting smoking could be more effective.

It is known that smokers in the general population have an increased frequency of elevated rheumatoid factor (RF). Since 1987, we have known that cigarette smok ing is a risk factor for rheumatoid arthritis (RA), especially in men with RF-positive who are heavy smokers. Later, it could be verified that cigarette smoking is implicated in the pathogenesis of RA particularly when associated with antibodies, cyclic citrullinated peptides (ACPA) and the shared epitope. Smoking increases the risk of both subsets of RA with a more pronounced influence on the risk of ACPA positive RA. The polyclonal immune response against modified antigens in patients with RA is not exclusively citrulline-specific, carbamylation of antigens like vimentin could be involved in the pathogenesis of the disease. Thus, smoking is a risk factor for breaking tolerance to multiple autoantigens in RA.

In RA patients, cigarette smoking correlated with an increased risk of joint erosions, extra-articular manifestations and cardiovascular disease, in addition to the established effects of longstanding disease activity on the risk of myocardial infarction and stroke. 
Systemic lupus erythematosus (SLE) is also associated with smoking. A strong and specific connection of current smoking and $>10$ pack-years of smoking with dsDNA+ SLE has been observed. In patients with SLE, smoking exposure has deleterious effects on lupus morbidity and is related to cumulative chronic damage. In mucocutaneous SLE, smoking have a negative impact on the efficacy of belimumab and a 2 -fold decrease in the proportion of patients achieving cutaneous improvement with antimalarials.

Published data indicate that smoking has a dose-dependent impact on structural damage progression in Ankylosing Spondylitis.

In a large UK cohort, smoking was positively associated with the risk of psoriatic arthritis in the general population, but negatively associated among patients with psoriasis. In addition, smokers with psoriatic arthritis had a poorer response to TNFi's compared to non-smokers.

Regarding osteoporosis, smoking has been identified as a predictor of ten-year fracture risk in men and women, largely independent of the densitometric results. This finding has eventually lead to incorporation of this risk factor into FRAX ${ }^{\circledR}$. The impact of smoking on bone status is mainly associated with the number of smoking years.

In a few reports, it has been observed an association between smoking and carpal tunnel syndrome and inflammatory bowel disease. Finally, current smoking in patients with fibromyalgia is associated with greater pain, possibly as a function of depression.

Disclosure of Interests: Antonio Naranjo Grant/research support from: Amgen, Consultant for: UCB, Speakers bureau: Amgen, UCB

DOI: 10.1136/annrheumdis-2019-eular.8546

\section{SP0089 CONSEQUENCES OF SMOKING CESSATION IN RHEUMATOLOGY}

Helen Harris. Western General Hospital, NHS Lothian, Rheumatology, Edinburgh, United Kingdom

Background: Cigarette smoking is a risk factor for many autoimmune and inflammatory conditions including rheumatoid arthritis (RA), psoriasis and psoriatic arthritis ( $\mathrm{PsO} \mathrm{PsA}$ ), is linked with active skin disease in patients with systemic lupus erythematosis (SLE) and poorer outcomes in patients with ankylosing spondylitis (AS) and systemic sclerosis (SSc). In addition there are well established risks of cardiovascular and respiratory diseases and increased risk of at least 13 cancers in patients that smoke [1]. Patients continuing to smoke after RA is diagnosed often fail to respond or require higher doses of immunosuppressive medications [2]. When arthritis is in remission and biologic dose reduction is attempted, patients that continue to smoke are more likely to have a flare of arthritis after dose reduction [3]. Patients that quit smoking can reduce or stop immunosuppressive medications without experiencing a flare [4]. Smokers have higher rates of infection than non-smokers; immunosuppressive therapy has an increased risk of infection and smoking cessation when starting treatment such as methotrexate or anti-TNF inhibitors can reduce the risk of infection [5]. The five top reasons to deliver brief smoking cessation advice to rheumatology patients are: 1. Improve chances of response to therapy. 2. Reduce infective risks of immunosuppressive therapy. 3. Improve success of medication dose reduction. 4. Reduce cardiovascular and respiratory diseases and cancer risks. 5. Reduce mortality.

Objectives: To demonstrate how brief smoking cessation advice can be delivered in rheumatology outpatient clinics and to show the consequences that this has had for patients, the rheumatology team and the wider healthcare system.

Methods: Delivering a brief intervention on smoking cessation by saying to smokers "Do you know the best way to quit? Use Champix (Varenicline) or Nicotine Replacement Therapy" is an evidence based way to motivate a quit attempt that takes 30 seconds to deliver. A 50 minute talk was presented to the rheumatology team of doctors, nurses and allied health professionals explaining this method of delivering brief advice on smoking cessation. We have made brief advice easy to deliver in the rheumatology clinic by creating a pathway for referral of smokers to the community pharmacist and smoking cessation nurse. A questionnaire has been administered to the rheumatology team to explore their attitudes and beliefs about delivering smoking cessation advice in the rheumatology clinic.

Results: The referral figures for the smoking cessation pathway will be presented. The consequences of delivering brief advice on smoking cessation for the patients, rheumatology team and healthcare system will be reported.

Conclusion: This presentation will show that helping rheumatology smokers to quit is achievable and should be considered an essential part of the rheumatology outpatient consultation.

\section{REFERENCES:}

[1] Arnson Y, Shoenfeld $Y$, Amital H. Effects of tobacco smoke on immunity, inflammation and autoimmunity. Journal of Autoimmunity 34 (2010) J258eJ265.
[2] Harris H, Tweedie F, White M, Samson K. How to persuade patients with Rheumatoid Arthritis to quit smoking. J Rheumatol. 2016 Apr;43(4):691-8. doi: 10.3899/jrheum.141368. Epub 2016 Feb 15.

[3] Gibson J, Harris H, McLaren J. Dose reduction of biologic therapies. EULAR OP0161 June 2012

[4] Andersson M, Bergman S et al, for the BARFOT Study Group $\S$ The Effect of Stopping Smoking on Disease Activity in Rheumatoid Arthritis (RA). Data from BARFOT, a Multicenter Study of Early RA. Open Rheumatol J. 2012; 6: 303-309.

[5] Arcavi L, Benowitz NL. Cigarette smoking and infection. Arch Intern Med. 2004 Nov 8;164(20):2206-16.

Disclosure of Interests: None declared

DOI: 10.1136/annrheumdis-2019-eular.8493

\section{SP0090 INTENSIVE SMOKING CESSATION INTERVENTION}

Ida Kristiane Roelsgaard. Rigshospitalet, Copenhagen Center for Arthritis Research (COPECARE), Center for Rheumatology and Spine Diseases, Glostrup, Denmark

Smokers with an inflammatory joint disease (IJD) experience poorer healthrelated quality of life compared to non-smokers with IJD (1). Smoking may exacerbate the symptoms of the disease and cause a reduced response to antirheumatic treatment (2-4). Smoking cessation interventions have traditionally been designed for people without chronic diseases (5). The literature on smoking cessation interventions to people with IJDs is limited.

Initially, this lecture will focus on "state of the art" regarding smoking cessation interventions to people with IJD.

Firstly, results from a systematic review will be presented. Randomised controlled trials (RCT) were included if they tested any form of smoking cessation intervention for adult daily smokers diagnosed with an IJD (6).

Secondly, the lecture will focus on various interventions for smoking cessation e. g. brief advice, behavioural interventions, motivational interviewing and nicotine replacement therapy.

Finally, principles for an ongoing RCTs where daily smokers with rheumatoid arthritis (RA) are randomised to either an intervention group or to a control group will be presented (7). The intervention consists of motivational counselling combined with nicotine replacement therapy. One patient research partner with RA (former smoker) has been involved with all phases of the study.

The above indicate that smoking cessation may positively impact not only clinical outcomes but also patient reported outcomes. However, a limited number of studies on smoking cessation interventions and the effect of smoking cessation on clinical and patient reported outcomes to people with IJD have been published. The knowledge is therefore limited, and health professionals should focus on this subject to proactively support people with IJDs who wish to quit smoking.

\section{REFERENCES}

[1] Bremander A, Jacobsson LT, Bergman S, Haglund E, Lofvendahl S, Petersson IF. Smoking is associated with a worse self-reported health status in patients with psoriatic arthritis: data from a Swedish populationbased cohort. Clinical rheumatology. 2015;34(3):579-83.

[2] Chang K, Yang SM, Kim SH, Han KH, Park SJ, Shin JI. Smoking and rheumatoid arthritis. International journal of molecular sciences. 2014;15 (12):22279-95.

[3] Abhishek A, Butt S, Gadsby K, Zhang W, Deighton CM. Anti-TNF-alpha agents are less effective for the treatment of rheumatoid arthritis in current smokers. Journal of clinical rheumatology : practical reports on rheumatic \& musculoskeletal diseases. 2010;16(1):15-8.

[4] Soderlin MK, Petersson IF, Geborek P. The effect of smoking on response and drug survival in rheumatoid arthritis patients treated with their first antiTNF drug. Scandinavian journal of rheumatology. 2012;41(1):1-9.

[5] Gritz ER, Vidrine DJ, Fingeret MC. Smoking cessation a critical component of medical management in chronic disease populations. American journal of preventive medicine. 2007;33(6 Suppl):S414-22.

[6] Roelsgaard IK, Esbensen BA, Østergaard M, Rollefstad S, Semb AG Christensen $\mathrm{R}$, et al. Smoking cessation intervention for reducing disease activity in chronic autoimmune inflammatory joint diseases. Cochrane Database of Systematic Reviews. 2018(2).

[7] Roelsgaard IK, Thomsen T, Ostergaard M, Christensen R, Hetland ML, Jacobsen $\mathrm{S}$, et al. The effect of an intensive smoking cessation intervention on disease activity in patients with rheumatoid arthritis: study protocol for a randomised controlled trial. Trials. 2017;18(1):570.

Disclosure of Interests: None declared

DOI: 10.1136/annrheumdis-2019-eular.8573 